\title{
Recent Advances in Drug Therapy for Parkinson's Disease
}

\author{
Hidetomo Murakami ${ }^{1}$, Tomotaka Shiraishi ${ }^{1}$, Tadashi Umehara ${ }^{1}$, \\ Shusaku Omoto ${ }^{2}$ and Yasuyuki Iguchi ${ }^{1}$
}

\begin{abstract}
:
Parkinson's disease (PD) is a neurodegenerative disease manifesting with motor and non-motor symptoms. Current treatment mainly relies on medication as a symptomatic therapy modulating neurotransmitters. Dopamine replacement therapy has been established, and levodopa is the gold standard for treatment of PD. However, the emergence of motor complications, such as a wearing-off phenomenon, is a clinical problem. Both primary symptoms and motor complications have been targets for the development of treatments for PD. Recent progression in the management of motor complications is supported by newly developed agents and advances in device and formulation technology to deliver drugs continuously. Elucidation of the pathophysiology of PD and the development of disease-modifying therapy that affects the underlying fundamental pathophysiology of the disease are also progressing. In this review, we introduce current knowledge on developments concerning medications for patients with PD.
\end{abstract}

Key words: Parkinson's disease, symptomatic therapy, motor fluctuation, continuous dopaminergic stimulation, non-dopaminergic drugs, disease-modifying therapy

(Intern Med Advance Publication)

(DOI: 10.2169/internalmedicine.8940-21)

\section{Introduction}

Parkinson's disease (PD) is a neurodegenerative disease manifesting with motor symptoms of akinesia/bradykinesia, tremor, and muscle rigidity as a triad, together with various non-motor symptoms. The number of patients with PD has increased rapidly with the aging of the population, and this is now referred to as a Parkinson pandemic.

Advances in treatment are anticipated, but current approaches mainly rely on drug therapy as symptomatic therapy modulating neurotransmitters in the brain. Degeneration of nigrostriatal dopaminergic neurons is the most common pathological finding in PD. Therefore, dopamine replacement therapy is the most fundamental treatment for PD patients. However, dopaminergic treatment for several years results in complications, such as the wearing-off phenomenon, which has prompted the development of symptomatic therapies. In the first section of this review, we introduce current drugs used for the symptomatic treatment of PD.

The pathophysiology of PD, such as the cause of neuro- nal damage, is becoming increasingly clear. Thus, the development of disease-modifying therapy that affects the underlying pathophysiology of the disease is ongoing. In the second section of the review, we introduce candidate diseasemodifying drugs currently in phase 2 or more advanced clinical trials.

\section{Symptomatic therapy}

Dopamine deficiency in the striatum of PD patients was first reported in the 1960s, and levodopa was developed as a drug that improves both the symptoms and life prognosis of patients (1). This drug is still the gold standard for treating PD. However, by the mid-1970s, it became apparent that motor complications, such as a wearing-off phenomenon, appear after using levodopa for several years (2). To date, reduction of these complications has been a focus of drug development for PD. To compensate for the shortcomings of levodopa, drugs with various mechanism of action have been developed. These include dopamine agonists that stimulate neuronal dopamine receptors, monoamine oxidase-

${ }^{1}$ Department of Neurology, The Jikei University School of Medicine, Japan and ${ }^{2}$ Department of Neurology, The Jikei University Katsushika Medical Center, Japan

Received: October 30, 2021; Accepted: December 8, 2021; Advance Publication by J-STAGE: February 1, 2022

Correspondence to Dr. Hidetomo Murakami, hidneu@jikei.ac.jp 
B (MAO-B) inhibitors that inhibit metabolism of dopamine in the brain, and catechol-O-methyl transferase (COMT) inhibitors that inhibit degradation of levodopa in the periphery. In this section of this review, we introduce drugs currently being used for symptomatic treatment of PD.

\section{Levodopa}

\section{1) Problems with current levodopa treatment}

Levodopa is the most reliable anti-PD drug for improvement of motor symptoms. However, the half-life of levodopa in blood is short (about $90 \mathrm{~min}$ ), which causes fluctuations in blood levels that result in changes in clinical symptoms in the advanced stage, manifesting as the wearing-off phenomenon. Thus, the development of levodopa therapy with a longer half-life using a different route of administration or formulation is being examined.

Neurotoxicity of levodopa was also suggested in the 1990 $\mathrm{s}$, which decreased the use of the drug for some time. However, it is now clear that neurotoxicity does not occur at the dose used in clinical practice, and the use of levodopa has since been reestablished. This return to use of levodopa is also partially due to the influence of advances in device and formulation technology that allow for continuous dopaminergic stimulation (CDS) with levodopa, as shown by the following representative formulations.

\section{2) Levodopa/carbidopa intestinal gel}

To overcome the short half-life of levodopa and improve motor symptoms during daytime activity, levodopa/carbidopa intestinal gel (LCIG) was developed. The drug is continuously infused into the upper jejunum through gastrostomy during daytime activities. LCIG was approved in Japan in 2016. In a study in East Asian patients with $\geq 3 \mathrm{~h}$ off-time per day, use of LCIG shortened the off-time by $4-5 \mathrm{~h}$ and extended the on-time without harmful dyskinesia by 5-6 h (3).

\section{3) Sustained release preparation of levodopa}

A sustained release capsule preparation (IPX066) packed with levodopa/carbidopa beads with a variety of fast to slow rates of dissolution in the gastrointestinal tract was developed to maintain the blood levodopa level longer than that using immediate release tablets. In a phase III study, the offtime was significantly shortened by IPX066 compared to immediate release tablets (4). Use of IPX066 has already been approved in western countries, but not in Japan.

\section{4) Levodopa inhalant}

A levodopa inhalant (CVT-301) has been approved in the US, but not in Japan, as a rescue medication during offtime. Levodopa is inhaled and rapidly absorbed from the lung. In a phase III study of inhalation of an 84-mg capsule, containing $42 \mathrm{mg}$ of levodopa, motor symptoms during offtime tended to be improved after $10 \mathrm{~min}$ and were significantly improved after $30 \mathrm{~min}$, compared with those before inhalation (5).

\section{5) Other levodopa formulations under development}

Levodopa formulations currently under development include an Accordion Pill ${ }^{\circledR}$ capsule utilizing a drug delivery system with a biodegradable polymeric film. The capsule is loaded with a folded multilayer film including levodopa/carbidopa. The ingested capsule dissolves in the stomach, and the folded film opens into a sheet shape and stays in the stomach for a maximum of $12 \mathrm{~h}$. Gradual release of levodopa/carbidopa from the film maintains a stable blood level, and the film is dissolved in the intestine after completion of drug release (6). A continuous subcutaneous levodopa injection (ND0612), of which efficacy to reduce fluctuations in plasma concentrations was shown in a phase 2 study (7), for PD patients with off-time is also under development. Phase 3 studies of both formulations are underway as of July 2021.

\section{Monoamine oxidase-B inhibitors}

\section{1) Novel MAO-B inhibitors}

MAO-B inhibitors increase the amount and duration of action of dopamine through inhibition of dopamine metabolism by MAO-B in the brain. In Japan, selegiline was approved in 1998 and has now been used for more than 20 years, while rasagiline was approved in 2018 and safinamide in 2019 as new MAO-B inhibitors. Single-agent administration of rasagiline at a dose of $1 \mathrm{mg}$ /day improves motor symptoms in early-stage PD patients (8), and addition of 0.5 or $1 \mathrm{mg} /$ day rasagiline significantly shortens the off-time and improves motor symptoms in advanced stage PD patients with motor complications under oral levodopa treatment (9). Safinamide extends the on-time and decreases the off-time in PD patients with a wearing-off phenomenon not accompanied by problematic dyskinesia and also improves motor symptoms during on-time (10). Selegiline, rasagiline and safinamide have different characteristics (Table 1), so selecting the most appropriate MAO-B inhibitor is now feasible. For example, safinamide has non-dopaminergic activity, including inhibitory effects on sodium channels and glutamate release, indicating a probable inhibitory effect on dyskinesia (11). However, the accumulation of further knowledge concerning appropriate use of MAO-B inhibitors is required.

\section{2) Monotherapy with a MAO-B inhibitor for de novo} PD

Selegiline was initially developed as an antidepressant in the 1960s. Its utility for motor symptoms of PD was first reported in the mid-1970s, and selegiline has become used mainly as an adjuvant of levodopa (12). In Japan, selegiline was approved in 1998 for concomitant use with levodopa. Administration of selegiline without levodopa activates the patient's endogenous dopamine, and overseas studies conducted before the approval of selegiline in Japan suggested that improvement of motor symptoms was acquired with monotherapy in patients with early PD (13). In Japan, administration of selegiline without concomitant levodopa was approved for health insurance coverage in 2011, enabling monotherapy.

MAO-B inhibitors were initially described as an option for first-line treatment in the 2018 edition of the Japanese 
Table 1. Characteristics of MAO-B inhibitors.

\begin{tabular}{|c|c|c|c|}
\hline & Selegiline & Rasagiline & Safinamide \\
\hline Indication & $\begin{array}{l}\text { Patients at Yahr severity scale } \\
\text { I-IV for concomitant use of a } \\
\text { levodopa-containing preparation, } \\
\text { patients at Yahr severity scale } \\
\text { I-III without concomitant use }\end{array}$ & $\begin{array}{l}\text { PD patients (no limitation for } \\
\text { concomitant drugs or severity) }\end{array}$ & $\begin{array}{l}\text { Patients with a wearing-off } \\
\text { phenomenon under treatment } \\
\text { with levodopa }\end{array}$ \\
\hline Monotherapy & Possible & Possible & $\begin{array}{l}\text { Not possible (concomitant } \\
\text { use with levodopa) }\end{array}$ \\
\hline Dose & $2.5-10.0 \mathrm{mg} / \mathrm{day}, 4$ steps & $\begin{array}{l}1.0 \mathrm{mg} / \text { day }(0.5 \mathrm{mg} / \text { day if } \\
\text { liver disorder is present })\end{array}$ & 50 or $100 \mathrm{mg} /$ day \\
\hline Number of oral doses per day & $1-2$ & 1 & 1 \\
\hline MAO-B inhibition & Irreversible & Irreversible & Reversible \\
\hline Dopamine activity of metabolite & Present & Absent & Absent \\
\hline $\begin{array}{l}\text { Designation as a raw material } \\
\text { for stimulant }\end{array}$ & Yes & No & No \\
\hline
\end{tabular}

guidelines for treatment of PD. MAO-B inhibitors have beneficial effects in addition to direct improvement of motor symptoms. Thus, initiation of early treatment with selegiline delays the time at which levodopa becomes necessary, compared with placebo (14), and concomitant use of selegiline within six months after treatment initiation with levodopa improves long-term motor symptoms and keeps the required dose of levodopa at a low level (15). Reduced aggravation of activities of daily living (ADL) and gait by using selegiline or rasagiline from the early stage has also been reported (16). These findings suggest a neuroprotective effect of MAO-B inhibitors, but this has not been shown clinically. In the PD-MED study (17), the clinical course was investigated based on the drug used for early treatment. Patients treated without levodopa had a lower rate of dyskinesia during a maximum seven-year course than those who received levodopa, and there were also fewer motor complications in non-levodopa cases treated with MAO-B inhibitors compared to dopamine agonists (17).

MAO-B inhibitor monotherapy is useful, but its effects vary widely among cases. For example, we administered selegiline alone to 28 unmedicated patients with PD and observed a range of improvement in motor symptoms that varied from high to low among cases (18). Improvement of motor symptoms by MAO-B inhibitor monotherapy as firstline treatment may be poor and lead to the requirement for concomitant levodopa or a dopamine agonist in some cases, but use of a MAO-B inhibitor from the early stage may be significant for the long-term course. A combined formulation of rasagiline and pramipexole, which has an effect complementary to the MAO-B inhibitor, is currently being developed for first-line treatment of PD (19). Further discussion of the positioning of MAO-B inhibitors as first-line treatment is needed.

\section{COMT inhibitors}

Entacapone is a COMT inhibitor that promotes levodopa entry into the brain by inhibiting metabolism of levodopa by COMT in the periphery. Entacapone has been used for sev- eral years in Japan, and opicapone was approved as a second COMT inhibitor in 2020. In patients with PD with motor complications under treatment with oral levodopa, opicapone significantly shortened the off-time and extended the on-time without accompanying harmful dyskinesia at both doses of 25 and $50 \mathrm{mg}$ /day compared with placebo (20). The long duration of action of opicapone permits once-a-day administration that supports levodopa activity at all time points. In contrast, entacapone has a relatively short duration of action that requires the drug to be taken simultaneously with levodopa at each time point. Appropriate use based on the status of each patient and characteristics of the drugs is required.

\section{Dopamine agonists}

Many dopamine agonists came into practical use in the 1980 s to 1990 s. Since these drugs have longer half-lives than levodopa and a low incidence of motor complications after early treatment, dopamine agonists are recommended to deal with the wearing-off phenomenon in early treatment and the advanced stage. Ergot-derived dopamine agonists were first used, but the main dopamine agonists have changed to non-ergot-derived drugs, as a risk of fibrosis, such as valvular disease of the heart, was pointed out with the earlier drugs. Sustained release preparations of two nonergot-derived drugs, pramipexole and ropinirole, were produced with the aim of achieving CDS, and a patch capable of maintaining a stable blood level by once-a-day replacement has also been developed. In Japan, a rotigotine patch was approved in 2013 and a ropinirole patch was approved in 2019, increasing the options for dopamine agonists. The affinity for receptor subtypes varies among these agonists, and different clinical effects may be expected. Furthermore, an overnight switch between dopamine agonists is possible, whereas 2-week withdrawal is necessary when switching MAO-B inhibitors. This is useful for selecting the most appropriate drug for each patient, and the safety of switching has also been shown for the recently approved ropinirole patch (21). 
Other advances in dopamine agonists include approval by the FDA in 2020 of a sublingual film formulation of apomorphine that can be easily handled. Continuous subcutaneous injection of apomorphine has already been approved in western countries. Whereas, only subcutaneous injection is approved in Japan as a rescue medication during off-time. In addition, most dopamine agonists developed for PD treatment mainly stimulate the dopamine D2 receptor, but an agonist with affinity for the D1/D5 receptor, tavapadon, is now under development (22), with a phase III study underway as of July 2021.

\section{Adenosine A2A receptor inhibitors}

Istradefylline received the first approval worldwide in Japan in 2013 as an inhibitor of the adenosine A2A receptor in the indirect pathway in the relatively hyperfunctional state among patients with PD. The indication is for improvement of the wearing-off phenomenon in PD under treatment with a regimen including levodopa, but an off-time shortening effect has also been observed, and this use was approved by the US FDA in 2019.

\section{Amantadine sustained release}

Amantadine, which was introduced as an agent for type A influenza in the 1970s, inhibits dyskinesia by inhibition of the NMDA receptor. This drug was also approved by the FDA in 2017 in a sustained release formulation (amantadine $274 \mathrm{mg}$ capsule), after a demonstration of the efficacy of this formulation in the EASE LID study (23). Sustained release of amantadine also improves motor symptoms during off-time in patients with PD with motor complications under treatment with levodopa (24), and this indication was added by the FDA in 2021.

\section{Therapeutic drugs for psychosis}

PD may be accompanied by hallucinations and delusions, and aggravation of motor symptoms by drugs interfering with the dopaminergic system is a concern. Pimavanserin, an inverse agonist of the 5-HT2A receptor, was approved in the US in 2016 for the treatment of hallucinations and delusions related to neurologic manifestations in patients with PD.

\section{Disease-modifying therapy}

PD is a heterogeneous disease for which the pathophysiology is increasingly becoming understood. This includes dysfunction in mitochondria or lysosomes, formation of toxic aggregates of $\alpha$-synuclein, neuroinflammation, oxidative stress, and other issues. These events are all potential targets of disease-modifying therapy that affects the underlying fundamental pathophysiology of the disease. Numerous studies have attempted to identify medications to inhibit progression of PD, and new compounds are under development. Drugs already used to treat other conditions and related disorders in clinical practice are also being repurposed as agents to treat PD in a process referred to as 'drug repositioning'. Such repositioning of already approved drugs can save costs and time compared to the development of new drugs. In the second section of the review, we introduce candidate disease-modifying drugs currently in phase 2 or more advanced clinical trials. A summary of these drugs is shown in Table 2.

\section{1. $\alpha$-synuclein targeting therapy}

\section{1) Immunization for $\alpha$-synuclein}

$\alpha$-Synuclein is a 140 -amino acid protein that is encoded by the SNCA gene. The physiological role of $\alpha$-synuclein is unclear, but its aggregation is toxic for neurons (25). The $\alpha$ synuclein oligomer causes mitochondrial dysfunction, endoplasmic reticulum stress, proteostasis dysregulation, synaptic impairment, cell apoptosis and neuroinflammation (26). Cell-to-cell propagation of $\alpha$-synuclein through prion-like spread is considered to be a pathophysiology of PD, and this propagation may occur during secretion of $\alpha$-synuclein by exosome exocytosis and endocytosis. According to Braak's theory (27), the pathology of $\alpha$-synuclein aggregation is proposed to begin in the medulla and spread gradually to the brain. Therefore, removal of extracellular $\alpha$ synuclein may prevent progression of the pathology and/or clinical symptoms of PD.

Immunization with anti- $\alpha$-synuclein oligomer monoclonal antibodies is currently being explored. For passive immunization, BIIB054 (cinpanemab), a monoclonal antibody that binds to the oligomeric and fibrillary forms of $\alpha$-synuclein, showed good tolerability and safety in a phase 1 trial (28), but the development of BIIP054 was halted in 2021 because of a lack of efficacy in the primary outcome of improvement of motor symptoms in the phase 2 (SPARK) study. PRX002 (prasinezumab) is also a monoclonal antibody directed against aggregated $\alpha$-synuclein. The safety and tolerability of PRX002 have been shown in a phase 1 study (29) and PRX002 is currently in phase 2 trials for PD (PASADENA study and PADOVA study). As an active vaccine for $\alpha$ synuclein, PD01A, which mimics the C-terminal region of $\alpha$-synuclein, has shown safety and tolerability in PD patients (30), and the AFFiRiS Corporation stated in 2020 that a phase 2 trial of PD01A for PD was in preparation.

\section{2) Inhibitor of misfolding of $\alpha$-synuclein}

NPT200-11 inhibits misfolding of $\alpha$-synuclein and subsequently inhibits its accumulation. NPT200-11 (UCB0599) and related compounds were developed through structurebased drug-discovery that utilized dynamic molecular modeling to identify and target specific regions of the alphasynuclein protein critical for the formation of misfolded oligomers $(31,32)$. Experiments using transgenic mice overexpressing human wild-type $\alpha$-synuclein showed that NPT20011 reduced $\alpha$-synuclein pathology in the cortex, reduced associated neuroinflammation (astrogliosis), normalized striatal levels of the dopamine transporter (DAT) and improved the motor function (32). Currently, a phase 2 study of the effects of NPT-200 on the motor and cognitive function and 
Table 2. Candidate Disease Modifying Therapy for PD Currently in Phase 2 or More Advanced Clinical Trials.

\begin{tabular}{|c|c|c|c|c|}
\hline Drugs & $\begin{array}{c}\text { Trial number } \\
\text { (Abbreviation of trial name) } \\
\end{array}$ & Phase & Subjects & Primary outcome \\
\hline \multicolumn{5}{|c|}{$\alpha$-synuclein targeting therapy } \\
\hline \multicolumn{5}{|c|}{ Monoclonal antibody } \\
\hline \multirow[t]{2}{*}{$\begin{array}{l}\text { PRX002 } \\
\text { (prasinezumab) }\end{array}$} & $\begin{array}{l}\text { NCT03100149, BP39529, } \\
\text { EudraCT-2017-000087-15 } \\
\text { (PASADENA) }\end{array}$ & 2 & Early PD & MDS-UPDRS Part I, II, III \\
\hline & $\begin{array}{c}\text { NCT04777331, BN42358, } \\
\text { EudraCT-2020-004997-23 (PADOVA) }\end{array}$ & 2 & Early PD & MDS-UPDRS Part III \\
\hline \multicolumn{5}{|l|}{ Vaccines } \\
\hline PD01A & Not registered yet & 2 (in preparation) & PD & Unknown \\
\hline \multicolumn{5}{|c|}{ Inhibitor of misfolding of $\alpha$-synuclein } \\
\hline $\begin{array}{l}\text { NPT200-11 } \\
\text { (UCB0599) }\end{array}$ & $\begin{array}{c}\text { NCT04658186, PD0053, EudraCT- } \\
\text { 2020-003265-19 }\end{array}$ & 2 & Early PD & MDS-UPDRS Parts I, II, III \\
\hline \multicolumn{5}{|c|}{ Enhancer of $\beta$-glucocerebrosidase } \\
\hline LTI-291 & Not registered yet & 2 (in preparation) & PD & Unknown \\
\hline \multicolumn{5}{|c|}{ Gene therapy using adeno-associated virus 9} \\
\hline PR001 & NCT04127578, PRV-PD101 (PROPEL) & $1 / 2 \mathrm{a}$ & PD with GBA1mutation & $\begin{array}{l}\text { Severe adverse event } \\
\text { Immunogenicity of AAV9 in blood/CSF } \\
\text { Immunogenicity of Ccase in blood/CSF }\end{array}$ \\
\hline \multicolumn{5}{|l|}{ GLP-1 receptor agonists } \\
\hline Exenatide & NCT04232969, 18/0320 (Exnatide-PD3) & 3 & Mild to moderate PD & MDS-UPDRS Part III \\
\hline $\begin{array}{l}\text { Sustained release } \\
\text { Exenatide (PT320) }\end{array}$ & NCT04269642. PT320-201 & 2 & Early PD & MDS-UPDRS Part III \\
\hline Semaglutide & NCT03659682, 120262PARK (GIPD) & 2 & Newly diagnosed PD & MDS-UPDRS Part III \\
\hline Liraglutide & NCT02953665, U1111-1173-0106 & 2 & $\begin{array}{c}\text { PD diagnosed at least } 2 \\
\text { years }\end{array}$ & MDS-UPDRS, NMSS, MADRS-2 \\
\hline Lixisenatide & NCT03439943, RC31/16/8912 (LixiPark) & 2 & Early PD & MDS-UPDRS Part III \\
\hline NLY01 & NCT04154072, NLY01-PD-1 & 2 & PD & MDS-UPDRS Part II, III \\
\hline \multicolumn{5}{|l|}{$\begin{array}{l}\text { Neuroprotective drugs } \\
\text { c-ABL kinase inhibitor }\end{array}$} \\
\hline Radotinib & NCT04691661, RT51EP1902 & 2 & $\begin{array}{l}\text { Early PD without } \\
\text { symptomatic medication }\end{array}$ & $\begin{array}{c}\text { Incidence and severity of treatment } \\
\text { emergent AEs }\end{array}$ \\
\hline \multicolumn{5}{|l|}{ Antibiotics } \\
\hline Ceftriaxone & NCT03413384, BRICEFA20170414 & 2 & Mild to Moderate PDD & ADAS-Cog \\
\hline \multicolumn{5}{|l|}{ Sigma-1 receptor agonist } \\
\hline $\begin{array}{l}\text { Blarcamesine } \\
\text { (ANAVEX 2-73) }\end{array}$ & $\begin{array}{l}\text { NCT04575259, ANAVEX2-73-PDD- } \\
\text { EP-001 }\end{array}$ & 2 & PD with dementia & Number of participants with adverse events \\
\hline \multicolumn{5}{|l|}{ Iron chelators } \\
\hline Deferiprone & $\begin{array}{l}\text { NCT02655315, } \\
\text { EudraCT-2015-003679-31 } \\
\text { (FAIRPARKII) }\end{array}$ & 2 & Treatment naïve PD & Total MDS-UPDRS score \\
\hline \multicolumn{5}{|l|}{ Analog of CoQ10 } \\
\hline \multirow[t]{2}{*}{ Idebenone } & NCT04152655 (SEASEiPPD) & $2 / 3$ & $\begin{array}{l}\text { REM Sleep Behavior } \\
\text { Disorder }\end{array}$ & Diagnosis of PD \\
\hline & NCT03727295, SZDX-1 (ITEP) & 4 & Early PD & $\begin{array}{l}\text { MDS-UPDRS Part III, Hoehn and Yahr } \\
\text { scale, Olfactory function test, Anxiety and } \\
\text { depression scale }\end{array}$ \\
\hline \multicolumn{5}{|l|}{ Immunosuppressant } \\
\hline Azathioprine & $\begin{array}{l}\text { ISRCTN14616801, EudraCT- 2018- } \\
\text { 003089-14 (AZA-PD) }\end{array}$ & 2 & Early PD & MDS-UPDRS Part III \\
\hline \multicolumn{5}{|l|}{ Statin } \\
\hline Simvastatin & $\begin{array}{c}\text { NCT02787590, ISRCTN16108482, } \\
\text { EudraCT 2015-000148-40, } \\
\text { PDSTAT2015 }\end{array}$ & 2 & $\begin{array}{l}\text { PD with wearing-off } \\
\text { phenomenon }\end{array}$ & MDS-UPDRS Part III \\
\hline Lovastatin & NCT03242499, NCTRC201702 & 2 & Early PD & MDS-UPDRS Part III \\
\hline \multicolumn{5}{|l|}{ Mitochondrial enhancer } \\
\hline Ursodeoxycholic acid & $\begin{array}{l}\text { NCT03840005, STH18493, } \\
\text { EudraCT-2018-001887-46 (UP study) }\end{array}$ & 2 & Early PD & Number of adverse treatment reactions \\
\hline
\end{tabular}


DAT imaging findings in patients with early PD is ongoing.

\section{Enhancers of $\beta$-glucocerebrosidase}

\section{1) $\beta$-glucocerebrosidase in $P D$}

$\beta$-glucocerebrosidase (GBA) is a lysosomal enzyme that cleaves glucocerebroside into ceramide and glucose by hydrolysis. Genetic variants of GBA are associated with Gaucher disease and PD. Decreased GBA activity results in the accumulation of glucocerebroside in neurons, which mediates decreased lysosomal activity, formation of toxic $\alpha-$ synuclein oligomers and consequent higher risks of developing $\mathrm{PD}$, more severe disease and faster progression of the disease. Drugs that affect the GBA function are under development.

\section{2) Ambroxol}

Ambroxol is an expectorant that has been shown to improve GBA activity in cells carrying GBA mutations and lysosomal activity in cells from patients with GBA mutationlinked PD (33). In the AiM-PD study, a non-randomized and non-controlled study, treatment with ambroxol improved the motor function of PD patients with and without GBI-1 mutations (34). A phase 2 study of the effects of ambroxol on the cognitive and motor function and cerebrospinal fluid and MRI findings in PD is currently underway.

\section{3) PR001A}

PR001A is injected intrathecally as a gene-replacement therapy using adeno-associated virus 9 (AAV9) to deliver a functional copy of the GBA1 gene to the brain (35). A phase 1-2a open label trial of PR001A for patients with GBA-associated PD is currently being performed.

\section{4) LTI-291}

LTI-291 is an allosteric modulator of GBA that enhances its activity. The results of a phase 1 trial have been published and showed that LTI-291 is well tolerated (36). According to an announcement on October 1, 2020, on the company's homepage (https://www.bial.com/com/, accessed on September 19,2021), a phase 2 trial should be ready to start in 2021.

\section{5) Venglustat (GZ/SAR402671)}

Venglustat is a glucocerebroside synthase inhibitor designed to reduce production of glucosylceramide. This "substrate reduction therapy" inhibits an upstream enzyme to reduce pathogenic substrate accumulation and is expected to have therapeutic efficacy for PD with GBA mutations. However, a phase 2 trial of the efficacy of venglustat in PD patients with GBA mutations (MOVES-PD study) did not meet the primary or secondary endpoints. Thus, further follow-up was terminated in 2021.

\section{Medication with neuroprotective effects}

\section{1) Glucagon-like peptide 1 receptor agonists}

Glucagon-like peptide-1 (GLP-1) receptor agonists are used to treat type II diabetes mellitus. GLP-1 receptor stimulation has also been shown to protect dopaminergic neurons from neurodegeneration in PD model mice (37). The proposed mechanism involves enhanced mitochondrial biogenesis, suppression of microglial activation and inflammation, enhancement of autophagy and clearance of aggregated proteins (38). A phase 2 study of exenatide, a GLP-1 receptor agonist, showed efficacy for motor symptoms and a reduced rate of decline in nigrostriatal dopaminergic neurons using DAT imaging (39). A phase 3 trial to examine the disease-modifying effect of exenatide in PD is ongoing (40). Phase 2 trials of other GLP-1 receptor agonists, including semaglutide, liraglutide, lixisenatide, LNY01 and a sustained release form of exenatide (PT320), are also ongoing in PD patients.

\section{2) c-Abl inhibitor}

The protein Abelson (c-Abl) is a non-receptor tyrosine kinase that is activated by oxidative and cellular stress. cAbl plays a role in the pathogenesis of PD, including in the aggregation of $\alpha$-synuclein and formation of Lewy bodies, autophagic impairment, mitochondrial dysfunction, and activation of microglia (41). Therefore, inhibition of c-Abl may influence the pathogenesis of PD. Some c-Abl inhibitors are already approved for treatment of chronic myelogenous leukemia, and recent studies in PD model mice suggest that cAbl inhibitors may have neuroprotective effects (41). Clinical studies have shown that nilotinib increased the CSF level of homovanillic acid, a dopamine metabolite $(42,43)$, reduced that of $\alpha$-synuclein oligomers (42), and improved the motor and cognitive function, suggesting a diseasemodifying effect (44). However, another trial of nilotinib showed no improvement in motor symptoms of PD patients (45). Two other c-Abl inhibitors-K-0706, which is also under the development for chronic myeloid leukemia, and radotinib-are also in phase 2 clinical trials.

\section{3) Ceftriaxone}

Ceftriaxone is a widely used antibiotic that has exhibited neuroprotective functions in an animal model of PD with dementia (PDD) $(46,47)$. Based on these findings, a phase II study to investigate the efficacy and safety of ceftriaxone in patients with mild to moderate PDD is ongoing in Taiwan.

\section{4) Sigma-1 receptor agonist}

Sigma-1 receptor is a chaperone protein localized in the mitochondria-associated endoplasmic reticulum membrane. Activation of sigma-1 receptor has neuroprotective effects, such as modulation of toxic intracellular cascades involving calcium ions and anti-inflammatory effects as well as the elevation of neurotrophic growth factors (48). Agonists of sigma-1 receptor induce autophagy and increase proteostasis capacity (49) and are candidate therapeutic agents for neurodegenerative diseases (50), especially PDD (51). A phase 2 study of blarcamesine (ANAVEX 2-73), a sigma-1 receptor agonist, is ongoing in PDD patients.

\section{Anti-oxidative stress drugs}

\section{1) Iron chelators}

In PD patients, iron accumulates in neurons of the substantia nigra (52), and the accumulated intracellular iron has a neurotoxic effect due to increased reactive oxygen 
stress (53). Therefore, iron chelators may be effective for preventing neuronal damage in PD. A phase 2 trial in 22 patients with mild PD showed that deferiprone, an iron chelator, was able to improve motor symptoms and decrease iron concentrations in the dentate and caudate nuclei (54). In the FAIRPARK trial, patients who started deferiprone immediately showed a significantly better motor performance at 6 or 12 months than those who started 6 months later (55). A phase 2 study of the efficacy of deferiprone (FAIRPARK-II study) is currently underway in patients with PD.

\section{2) Analogs of coenzyme Q10}

Idebenone is an analog of the well-known antioxidant coenzyme Q10 (CoQ10) and has been shown to mitigate motor impairment and to increase the neuron survival in PD model animals (56). Clinical trials of idebenone for protection against the development of PD in patients with REM sleep behavior disorder (SEASEiPPD study) and therapeutic effects on motor and non-motor symptoms in patients with early PD (ITEP study) are ongoing.

\section{3) Myeloperoxidase inhibitors}

Oxidative stress is one of the implicated pathogeneses of PD. Myeloperoxidase (MPO) is a reactive oxygen generating enzyme, and MPO-immunoreactive cells are increased in brain regions affected by neurodegeneration in PD (57). Oxidative stress is associated with neuroinflammation and neural damage in PD, and inhibition of MPO may reduce oxidative stress, neuroinflammation and neuronal damage in PD patients. A phase 2 study of AZD3241 (verdiperstat), a MPO inhibitor, in PD patients showed a reduction in distribution of activated microglia using ${ }^{11} \mathrm{C}$-PBR28 positron emission tomography (58). Other clinical trials for PD were planned, but whether or not the further development of AZD 3241 for PD is underway is unclear.

\section{Anti-inflammatory agents and immunosuppres- sants}

\section{1) Non-steroidal anti-inflammatory drugs (NSAIDs)} and immunosuppressants

Dysregulated inflammatory and immune systems, in which activated astrocytes, microglia and peripheral immune cells as well as inflammatory cytokines are present, are also implicated in the etiology of PD $(59,60,61)$. Regular use of NSAIDs at baseline has been associated with a reduced risk of PD (62), and among NSAIDs, ibuprofen has been shown to have a particularly marked effect (63). A population-based case-control study of United States Medicare beneficiaries showed that the use of immunosuppressants, such as azathioprine, and corticosteroids was also associated with a reduced risk of emergence of PD (64). Therefore, anti-inflammatory drugs and immunosuppressants may have disease-modifying effects in PD. Among immunosuppressants, azathioprine, which reduces the proliferation of $\mathrm{B}$ and $\mathrm{T}$ cells via the inhibition of nucleic acid synthesis, is widely used in various immune-related disorders in clinical practice. A phase 2 randomized placebo-controlled, double-blind trial of the effects of azathioprine on progres- sion of motor and non-motor symptoms in early PD patients (AZA-PD study) is in preparation (65).

\section{2) Statins}

Statins are inhibitors of 3-hydroxy-3-methylglutaryl coenzyme A (HMG-CoA) reductase and are commonly used in clinical practice to treat dyslipidemia. These drugs have also been suggested to have anti-oxidative and anti-inflammatory effects $(66,67)$ and to reduce intraneuronal $\alpha$-synuclein aggregation (68). A population study showed that the continuation of lipophilic statin therapy was associated with a decreased incidence of PD compared to patients with discontinuation of statins (69). A recent trial showed that lovastatin treatment in patients with early-stage PD was associated with a trend of reduced exacerbation of motor symptoms (70). These findings suggest that statins are candidates for neuroprotective treatment for PD. A phase 2 trial examining the effect of simvastatin on PD with a wearing-off phenomenon is currently ongoing (71), and the effects of lovastatin on motor symptoms in early PD patients are being examined in a phase 2 trial.

\section{Recovery of the mitochondrial function}

Mitochondrial dysfunction is a pathogenesis of PD and believed to be a promising target for disease-modifying therapy (72). Mortiboys et al. showed that ursodeoxycholic acid, which has been used for the treatment of liver disease for over 30 years, improved the mitochondrial production of ATP in an in vitro study using parkin-mutant fibroblasts and LRRK2 $2^{\text {G2049S }}$ mutant fibroblasts (73). Ursodeoxycholic acid has also been shown to rescue the function of mitochondria in LRRS2 $2^{\text {G2019S }}$ carriers in vivo (74). Therefore, ursodeoxycholic acid may ameliorate the pathophysiology of PD by improving mitochondrial dysfunction. A phase 2 trial to ascertain the effect of ursodeoxycholic acid on mitochondrial activity, progression of motor symptoms and other effects in patients with PD is ongoing (75).

\section{Conclusion}

In this review, we focused on recent developments of symptomatic and disease-modifying therapy for patients with PD. The search for medications for PD has continued with treatment utilizing already existing drugs, as well as the development of new drugs. Levodopa is still the gold standard for PD, but the high prevalence of motor fluctuation with levodopa is a concern. Treatment options for motor fluctuation as symptomatic therapy are being developing with novel agents and advances in device and formulation technology. Disease-modifying therapy is not yet available in clinical practice, but progress in this area is likely as the pathophysiology of PD is further understood, and this approach may become practical in the near future.

The authors state that they have no Conflict of Interest (COI). 


\section{References}

1. Hoehn MM, Yahr MD. Parkinsonism: onset, progression and mortality. Neurology 17: 427-442, 1967.

2. Marsden CD, Parkes JD. "On-off" effects in patients with Parkinson's disease on chronic levodopa therapy. Lancet 1: 292-296, 1976.

3. Murata M, Mihara M, Hasegawa K, et al. Efficacy and safety of levodopa-carbidopa intestinal gel from a study in Japanese, Taiwanese, and Korean advanced Parkinson's disease patients. NPJ Parkinsons Dis 2: 16020, 2016.

4. Hauser RA, Hsu A, Kell S, et al. Extended-release carbidopalevodopa (IPX066) compared with immediate-release carbidopalevodopa in patients with Parkinson's disease and motor fluctuations: a phase 3 randomised, double-blind trial. Lancet Neurol 12: 346-356, 2013.

5. LeWitt PA, Hauser RA, Pahwa R, et al. Safety and efficacy of CVT-301 (levodopa inhalation powder) on motor function during off periods in patients with Parkinson's disease: a randomised, double-blind, placebo-controlled phase 3 trial. Lancet Neurol 18: 145-154, 2019.

6. Navon N. The Accordion Pill ${ }^{\circledR}$ : unique oral delivery to enhance pharmacokinetics and therapeutic benefit of challenging drugs. Ther Deliv 10: 433-442, 2019.

7. Giladi N, Gurevich T, Djaldetti R, et al. ND0612 (levodopa/carbidopa for subcutaneous infusion) in patients with Parkinson's disease and motor response fluctuations: A randomized, placebocontrolled phase 2 study. Parkinsonism Relat Disord 91: 139-145, 2021.

8. Hattori N, Takeda A, Takeda S, et al. Rasagiline monotherapy in early Parkinson's disease: A phase 3, randomized study in Japan. Parkinsonism Relat Disord 60: 146-152, 2019.

9. Hattori N, Takeda A, Takeda S, et al. Efficacy and safety of adjunctive rasagiline in Japanese Parkinson's disease patients with wearing-off phenomena: A phase $2 / 3$, randomized, double-blind, placebo-controlled, multicenter study. Parkinsonism Relat Disord 53: 21-27, 2018.

10. Hattori N, Tsuboi Y, Yamamoto A, Sasagawa Y, Nomoto M; ME 2125-3 Study Group. Efficacy and safety of safinamide as an addon therapy to L-DOPA for patients with Parkinson's disease: A randomized, double-blind, placebo-controlled, phase II/III study. Parkinsonism Relat Disord 75: 17-23, 2020.

11. Cattaneo C, Ferla RL, Bonizzoni E, Sardina M. Long-term effects of safinamide on dyskinesia in mid- to late-stage Parkinson's disease: a post-hoc analysis. J Parkinsons Dis 5: 475-481, 2015.

12. Birkmayer W, Riederer $P$, Ambrozi L, Youdim MB. Implications of combined treatment with 'Madopar' and L-deprenil in Parkinson's disease. A long-term study. Lancet 1: 439-443, 1977.

13. Csanda E, Tárczy M. Clinical evaluation of deprenyl (selegiline) in the treatment of Parkinson's disease. Acta Neurol Scand Suppl 95: 117-122, 1983.

14. Parkinson Study Group. Effects of tocopherol and deprenyl on the progression of disability in early Parkinson's disease. N Engl J Med 328: 176-183, 1993.

15. Larsen JP, Boas J, Erdal JE. Does selegiline modify the progression of early Parkinson's disease? Results from a five-year study. The Norwegian-Danish Study Group. Eur J Neurol 6: 539-547, 1999.

16. Hauser RA, Li R, Pérez A, et al. Longer duration of MAO-B inhibitor exposure is associated with less clinical decline in Parkinson's disease: an analysis of NET-PD LS1. J Parkinsons Dis 7: 117-127, 2017

17. Gray R, Ives N, et al.; PD Med Collaborative Group. Long-term effectiveness of dopamine agonists and monoamine oxidase B inhibitors compared with levodopa as initial treatment for Parkinson's disease (PD MED): a large, open-label, pragmatic random- ised trial. Lancet 384: 1196-1205, 2014.

18. Murakami H, Nohara T, Uchiyama M, et al. Accumulation of ${ }^{123} \mathrm{I}-$ ioflupane is a useful marker of the efficacy of selegiline monotherapy in drug-naïve Parkinson's disease. Front Aging Neurosci 9: 321, 2017.

19. Olanow CW, Kieburtz K, Leinonen $\mathrm{M}$, et al. A randomized trial of a low-dose Rasagiline and Pramipexole combination (P2B001) in early Parkinson's disease. Mov Disord 32: 783-789, 2017.

20. Takeda A, Takahashi R, Tsuboi Y, et al. Randomized, Controlled Study of Opicapone in Japanese Parkinson's Patients with Motor Fluctuations. Mov Disord 36: 415-423, 2021.

21. Mochizuki H, Hattori N, Hasegawa K, et al. Long-term study of ropinirole patch in Parkinson's disease patients with/without basal L-dopa. Parkinsonism Relat Disord 83: 105-109, 2021.

22. Riesenberg R, Werth J, Zhang Y, Duvvuri S, Gray D. PF06649751 efficacy and safety in early Parkinson's disease: a randomized, placebo-controlled trial. Ther Adv Neurol Disord 13: 1756286420911296, 2020.

23. Pahwa R, Tanner CM, Hauser RA, et al. ADS-5102 (Amantadine) Extended-Release Capsules for Levodopa-Induced Dyskinesia in Parkinson Disease (EASE LID Study): A Randomized Clinical Trial. JAMA Neurol 74: 941-949, 2017.

24. Hauser RA, Mehta SH, Kremens D, Chernick D, Formella AE. Effects of Gocovri (Amantadine) Extended-Release Capsules on Motor Aspects of Experiences of Daily Living in People with Parkinson's Disease and Dyskinesia. Neurol Ther in press. Epub ahead of print.

25. Ono K. The Oligomer Hypothesis in $\alpha$-Synucleinopathy. Neurochem Res 42: 3362-3371, 2017.

26. Du XY, Xie XX, Liu RT. The Role of $\alpha$-Synuclein Oligomers in Parkinson's Disease. Int J Mol Sci 21: 8645, 2020.

27. Braak H, Del Tredici K, Rüb U, de Vos RA, Jansen Steur EN, Braak E. Staging of brain pathology related to sporadic Parkinson's disease. Neurobiol Aging 24: 197-211, 2003.

28. Brys M, Fanning L, Hung S, et al. Randomized phase I clinical trial of anti- $\alpha$-synuclein antibody BIIB054. Mov Disord 34: 1154$1163,2019$.

29. Jankovic J, Goodman I, Safirstein B, et al. Safety and Tolerability of Multiple Ascending Doses of PRX002/RG7935, an Anti- $\alpha$ Synuclein Monoclonal Antibody, in Patients With Parkinson Disease: A Randomized Clinical Trial. JAMA Neurol 75: 1206-1214, 2018.

30. Volc D, Poewe W, Kutzelnigg A, et al. Safety and immunogenicity of the $\alpha$-synuclein active immunotherapeutic PD01A in patients with Parkinson's disease: a randomised, single-blinded, phase 1 trial. Lancet Neurol 19: 591-600, 2020.

31. Wrasidlo W, Tsigelny IF, Price DL, et al. A de novo compound targeting $\alpha$-synuclein improves deficits in models of Parkinson's disease. Brain 139: 3217-3236, 2016.

32. Price DL, Koike MA, Khan A, et al. The small molecule alphasynuclein misfolding inhibitor, NPT200-11, produces multiple benefits in an animal model of Parkinson's disease. Sci Rep 8: 16165,2018

33. McNeill A, Magalhaes J, Shen C, et al. Ambroxol improves lysosomal biochemistry in glucocerebrosidase mutation-linked Parkinson disease cells. Brain 137: 1481-1495, 2014.

34. Mullin S, Smith L, Lee K, et al. Ambroxol for the Treatment of Patients With Parkinson Disease With and Without Glucocerebrosidase Gene Mutations: A Nonrandomized, Noncontrolled Trial. JAMA Neurol 77: 427-434, 2020.

35. Abeliovich A, Hefti F, Sevigny J. Gene Therapy for Parkinson's Disease Associated with GBA1 Mutations. J Parkinsons Dis 11: S 183-S188, 2021.

36. den Heijer JM, Kruithof AC, van Amerongen G, et al. A randomized single and multiple ascending dose study in healthy volunteers of LTI-291, a centrally penetrant glucocerebrosidase activa- 
tor. Br J Clin Pharmacol 87: 3561-3573, 2021.

37. Li Y, Perry T, Kindy MS, et al. GLP-1 receptor stimulation preserves primary cortical and dopaminergic neurons in cellular and rodent models of stroke and Parkinsonism. Proc Natl Acad Sci U S A 106: 1285-1290, 2009.

38. Athauda D, Foltynie T. The glucagon-like peptide 1 (GLP) receptor as a therapeutic target in Parkinson's disease: mechanisms of action. Drug Discov Today 21: 802-818, 2016.

39. Athauda D, Maclagan K, Skene SS, et al. Exenatide once weekly versus placebo in Parkinson's disease: a randomised, double-blind, placebo-controlled trial. Lancet 390: 1664-1675, 2017.

40. Vijiaratnam N, Girges C, Auld G, et al. Exenatide once weekly over 2 years as a potential disease-modifying treatment for Parkinson's disease: protocol for a multicentre, randomised, double blind, parallel group, placebo controlled, phase 3 trial: The 'Exenatide-PD3' study. BMJ Open 11: e047993, 2021.

41. Abushouk AI, Negida A, Elshenawy RA, et al. C-Abl Inhibition; A Novel Therapeutic Target for Parkinson's Disease. CNS Neurol Disord Drug Targets 17: 14-21, 2018.

42. Pagan FL, Hebron ML, Wilmarth B, et al. Nilotinib Effects on Safety, Tolerability, and Potential Biomarkers in Parkinson Disease: A Phase 2 Randomized Clinical Trial. JAMA Neurol 77: 309-317, 2020.

43. Pagan FL, Hebron ML, Wilmarth B, et al. Pharmacokinetics and pharmacodynamics of a single dose Nilotinib in individuals with Parkinson's disease. Pharmacol Res Perspect 7: e00470, 2019.

44. Pagan F, Hebron M, Valadez EH, et al. Nilotinib Effects in Parkinson's disease and Dementia with Lewy bodies. J Parkinsons Dis 6: 503-517, 2016

45. Simuni T, Fiske B, Merchant K, et al. Efficacy of Nilotinib in Patients With Moderately Advanced Parkinson Disease: A Randomized Clinical Trial. JAMA Neurol 78: 312-320, 2021.

46. Hsu CY, Hung CS, Chang HM, Liao WC, Ho SC, Ho YJ. Ceftriaxone prevents and reverses behavioral and neuronal deficits in an MPTP-induced animal model of Parkinson's disease dementia. Neuropharmacology 91: 43-56, 2015.

47. Hsieh MH, Meng WY, Liao WC, et al. Ceftriaxone reverses deficits of behavior and neurogenesis in an MPTP-induced rat model of Parkinson's disease dementia. Brain Res Bull 132: 129-138, 2017.

48. Ruscher K, Wieloch $\mathrm{T}$. The involvement of the sigma-1 receptor in neurodegeneration and neurorestoration. J Pharmacol Sci 127: 3035, 2015.

49. Christ MG, Huesmann H, Nagel H, Kern A, Behl C. Sigma-1 Receptor Activation Induces Autophagy and Increases Proteostasis Capacity In Vitro and In Vivo. Cells 8: 211, 2019.

50. Su TP, Su TC, Nakamura Y, Tsai SY. The Sigma-1 Receptor as a Pluripotent Modulator in Living Systems. Trends Pharmacol Sci 37: 262-278, 2016.

51. Sun C, Armstrong MJ. Treatment of Parkinson's Disease with Cognitive Impairment: Current Approaches and Future Directions. Behav Sci (Basel) 11: 54, 2021.

52. Oakley AE, Collingwood JF, Dobson J, et al. Individual dopaminergic neurons show raised iron levels in Parkinson disease. Neurology 68: 1820-1825, 2007.

53. Ward RJ, Zucca FA, Duyn JH, Crichton RR, Zecca L. The role of iron in brain ageing and neurodegenerative disorders. Lancet Neurol 13: 1045-1060, 2014

54. Martin-Bastida A, Ward RJ, Newbould R, et al. Brain iron chelation by deferiprone in a phase 2 randomised double-blinded placebo controlled clinical trial in Parkinson's disease. Sci Rep 7: 1398, 2017.

55. Devos D, Moreau C, Devedjian JC, et al. Targeting chelatable iron as a therapeutic modality in Parkinson's disease. Antioxid Redox Signal 21: 195-210, 2014.

56. Avcı B, Günaydın C, Güvenç T, Yavuz CK, Kuruca N, Bilge SS.
Idebenone Ameliorates Rotenone-Induced Parkinson's Disease in Rats Through Decreasing Lipid Peroxidation. Neurochem Res 46: 513-522, 2021.

57. Gellhaar S, Sunnemark D, Eriksson H, Olson L, Galter D. Myeloperoxidase-immunoreactive cells are significantly increased in brain areas affected by neurodegeneration in Parkinson's and Alzheimer's disease. Cell Tissue Res 369: 445-454, 2017.

58. Jucaite A, Svenningsson P, Rinne JO, et al. Effect of the myeloperoxidase inhibitor AZD3241 on microglia: a PET study in Parkinson's disease. Brain 138: 2687-700, 2015.

59. Hirsch EC, Hunot S. Neuroinflammation in Parkinson's disease: a target for neuroprotection? Lancet Neurol 8: 382-397, 2009.

60. Tansey MG, Romero-Ramos M. Immune system responses in Parkinson's disease: Early and dynamic. Eur J Neurosci 49: 364383, 2019.

61. MacMahon Copas AN, McComish SF, Fletcher JM, Caldwell MA. The Pathogenesis of Parkinson's Disease: A Complex Interplay Between Astrocytes, Microglia, and T Lymphocytes? Front Neurol 12: 666737, 2021.

62. Chen H, Zhang SM, Hernán MA, et al. Nonsteroidal antiinflammatory drugs and the risk of Parkinson disease. Arch Neurol 60: 1059-1064, 2003.

63. Gao X, Chen H, Schwarzschild MA, Ascherio A. Use of ibuprofen and risk of Parkinson disease. Neurology 76: 863-869, 2011.

64. Racette BA, Gross A, Vouri SM, Camacho-Soto A, Willis AW, Searles Nielsen S. Immunosuppressants and risk of Parkinson disease. Ann Clin Transl Neurol 5: 870-875, 2018.

65. Greenland JC, Cutting E, Kadyan S, Bond S, Chhabra A, Williams-Gray $\mathrm{CH}$. Azathioprine immunosuppression and disease modification in Parkinson's disease (AZA-PD): a randomised double-blind placebo-controlled phase II trial protocol. BMJ Open 10: e040527, 2020.

66. Wang Q, Yan J, Chen X, et al. Statins: multiple neuroprotective mechanisms in neurodegenerative diseases. Exp Neurol 230: 2734, 2011

67. Wood WG, Eckert GP, Igbavboa U, Müller WE. Statins and neuroprotection: a prescription to move the field forward. Ann N Y Acad Sci 1199: 69-76, 2010.

68. Bar-On P, Crews L, Koob AO. Statins reduce neuronal alphasynuclein aggregation in in vitro models of Parkinson's disease. J Neurochem 105: 1656-1667, 2008.

69. Lee YC, Lin CH, Wu RM, et al. Discontinuation of statin therapy associates with Parkinson disease: a population-based study. Neurology 81: 410-416, 2013.

70. Lin $\mathrm{CH}$, Chang $\mathrm{CH}$, Tai $\mathrm{CH}$, et al. A Double-Blind, Randomized, Controlled Trial of Lovastatin in Early-Stage Parkinson's Disease. Mov Disord 36: 1229-1237, 2021.

71. Carroll CB, Webb D, Stevens KN, et al. Simvastatin as a neuroprotective treatment for Parkinson's disease (PD STAT): protocol for a double-blind, randomised, placebo-controlled futility study. BMJ Open 9: e029740, 2019.

72. Schapira AH, Olanow CW, Greenamyre JT, Bezard E. Slowing of neurodegeneration in Parkinson's disease and Huntington's disease: future therapeutic perspectives. Lancet 384: 545-555, 2014.

73. Mortiboys H, Aasly J, Bandmann O. Ursocholanic acid rescues mitochondrial function in common forms of familial Parkinson's disease. Brain 136: 3038-3050, 2013.

74. Mortiboys H, Furmston R, Bronstad G, Aasly J, Elliott C, Bandmann O. UDCA exerts beneficial effect on mitochondrial dysfunction in LRRK2(G2019S) carriers and in vivo. Neurology 85: 846-852, 2015.

75. Payne T, Sassani M, Buckley E, et al. Ursodeoxycholic acid as a novel disease-modifying treatment for Parkinson's disease: protocol for a two-centre, randomised, double-blind, placebo-controlled trial, The 'UP' study. BMJ Open 10: e038911, 2020. 
The Internal Medicine is an Open Access journal distributed under the Creative

by-nc-nd/4.0/). Commons Attribution-NonCommercial-NoDerivatives 4.0 International License. To view the details of this license, please visit (https://creativecommons.org/licenses/

(C) The Japanese Society of Internal Medicine Intern Med Advance Publication 